Review

\title{
Defining the Role of Tyrosine Kinase Inhibitors in Early Stage Non-Small Cell Lung Cancer
}

\author{
Sofia Lampaki ${ }^{1}$, George Lazaridis ${ }^{2}$, Konstantinos Zarogoulidis ${ }^{1}$, Ioannis Kioumis ${ }^{1}$, Antonis Papaiwannou ${ }^{1}$, \\ Katerina Tsirgogianni ${ }^{1}$, Anastasia Karavergou ${ }^{1}$, Theodora Tsiouda ${ }^{3}$, Vasilis Karavasilis ${ }^{3}$, Lonny Yarmus ${ }^{4}$, \\ Kaid Darwiche ${ }^{5}$, Lutz Freitag5, Antonios Sakkas ${ }^{6}$, Angeliki Kantzeli7, Sofia Baka ${ }^{8}$, Wolfgang \\ Hohenforst-Schmidt ${ }^{9}$ and Paul Zarogoulidis ${ }^{1}$ \\ 1. Pulmonary Department-Oncology Unit, "G. Papanikolaou” General Hospital, Aristotle University of Thessaloniki, Thessaloniki, Greece, \\ 2. Department of Medical Oncology, "G. Papageorgiou" University Hospital, Thessaloniki, Greece, Nea Eukarpia. \\ 3. Pulmonary Department, "Theageneio Anticancer" Hospital, Thessaloniki, Greece, Alexander Simeonidi 2 \\ 4. Division of Pulmonary and Critical Care Medicine, Johns Hopkins University, Baltimore, U.S.A. \\ 5. Department of Interventional Pulmonology, Ruhrlandklinik, University Hospital Essen, University Duisburg-Essen, Essen, Germany. \\ 6. Department of Internal Medicine, "Evangelisches Krankenhaus", Mülheim, Germany \\ 7. Department of Internal Medicine, "Helios Klinikum", Wuppertal, Germany \\ 8. Oncology Department, "Interbalkan" European Medical Center, Thessaloniki, Greece \\ 9. Medical Clinic I, "Fuerth" Hospital, University of Erlangen, Fuerth, Germany
}

$\square$ Corresponding author: Paul Zarogoulidis, M.D, Ph. D, Pulmonary Department-Oncology Unit, “G. Papanikolaou” General Hospital, Aristotle University of Thessaloniki, Thessaloniki, Greece. Fax: 00302310992424; Mobile: 00306977271974; E-mail: pzarog@hotmail.com

(c) 2015 Ivyspring International Publisher. Reproduction is permitted for personal, noncommercial use, provided that the article is in whole, unmodified, and properly cited. See http://ivyspring.com/terms for terms and conditions.

Received: 2015.02.14; Accepted: 2015.04.14; Published: 2015.05.07

\begin{abstract}
Historical, the non-small cell lung cancer (NSCLC) was as a united disease entity and the chemotherapy to the metastatic cancer had limited results. Recent studies for the metastatic non-small cell lung cancer led to the ascertainment that the NSCLC does not constitute exclusively a disease entity, but different neoplasms guided from different molecular paths, different biological behavior and at extension requires different confrontation. Thus the new direction for the therapeutic approach of NSCLC is henceforth the most individualized approach based on the activated molecular paths of tumor. Distinct subtypes of NSCLC are driven by a specific genetic alteration, like EGFR, ALK, ROSI or BRAF mutations, and these genetic alterations are sensitized to the inhibition of specific oncogenic pathways. The benefit from the use of tyrosine kinase inhibitors in patients with EGFR mutations it was confirmed by six randomized studies of phase III that investigated the role of gefitinib, erlotinib and afatinib. In these studies the response rates vary in the impressive percentages from $55 \%$ to $86 \%$ and were connected with a remarkable median progression free survival of approximately 8 to 13 months, and with better quality of life compared to that of chemotherapy. In early stages NSCLC is needed the individualization of systemic treatment in order to reduce toxicity that is observed in the classic chemotherapy and to impact outcome. The role of EGFR TKI's has been evaluated in the adjuvant chemotherapy in early stage resected NSCLC. The data from these studies suggest that adjuvant TKI therapy might not increase the overall survival, but delay the recurrences. Prospective trials restricted to EGFR or ALK driven NSCLC subsets potentially offering the opportunity for a definitive answer in early disease adjuvant setting (ALCHEMIST) or as induction treatment before stage III chemo-radiotherapy (RTOG 1210/Alliance 31101), are ongoing. Ongoing prospective trials may offer the opportunity for a definitive answer of the role of tyrosine kinase inhibitors in induction treatment before chemo-radiotherapy or in early disease adjuvant therapy.
\end{abstract}

Key words: lung cancer,egfr, iressa, tarceva 


\section{Introduction}

Over the last years, billions have been spent and enormous efforts have been taken in clinical research of cancer. [1] Approximately before 10 years ago we had the introduction of tyrosine kinase inhibitors (TKI) into pharmacological anti-cancer therapy. [2] TKI's, according to their molecular mechanism of action, appertain to the group of targeted cancer medicines, in contrast to the classical or conventional cytostatics, represented by accurately fitting with biological structures. [3] Distinct subtypes of NSCLC are driven by a specific genetic alteration, like EGFR, ALK, ROS1 or BRAF mutations, and are thus sensitive to inhibition of the corresponding activated oncogenic pathway. [4]

The researchers consider the pathobiology of NSCLC to the forming of small molecules that target mutations of genes that play important roles in the metastatic disease. Testing of the mutations in epidermal growth factor receptor (EGFR), KRAS, and anaplastic lymphoma kinase (ALK) is the today's standard of care. $[5,6,7]$

On the cell surface of a substantial percentage of non small cell lung cancer cells expressed the EGFR. Initially the EGFR tyrosine kinase inhibitors (TKIs), the gefitinib (Iressa) and the erlotinib (Tarceva) had shown clinical activity and also biologic activity only in a subgroup of NSCLC. [8] From further research, the investigators demonstrate that it is possible to have highest response rates in patients with mutations to the exon 19 deletion, to the exon 21 L858R, and to the exon 18 G719X. [9] Contrariwise the exon 20 T790M mutation related with resistance to TKI therapy. [10] Generally EGFR mutations noticed more often in adenocarcinomas, in patients without prior history of smoking, and in females and more commonly in those of Asian origin. [11]

Gefitinib, erlotinib, and afatinib are used in patients with adenocarcinomas with known EGFR mutations.

KRAS mutations are found in about $25 \%$ of adenocarcinomas, and they are less frequent in Asian patients and more often in smokers. [12] Remarkably the patients with KRAS mutations appear to be resistant to EGFR-TKIs therapy and these patients who carry KRAS mutations have a poorer prognosis. [13, 14]

ALK rearrangements, like fusion between echinoderm microtubule-associated proteinlike 4 (EML4) and ALK are noticed in around $2-7 \%$ of patients with adenocacrinomas of the lung and more often in non or light smokers. Due to the fact that EGFR and ALK mutations are mutually exclusive, to the patients who carry ALK rearrangements are indicated treatment with an ALK inhibitor (crizotinib [Xalkori], or ceritinib [Zykadia]). [15, 16, 17]

The first EGFR-TKI was gefitinib, which was assessed in a phase III trial, in which the patients were not prospectively stratified according to mutation status. The results from this trial showed that there was not benefit to survival and only $10 \%$ of the patients showed response to this therapy. [18] In a meta-analysis of tissue samples found that in 8 of 9 patients who showed response carried EGFR mutations and in 7 patients who did not have response there was not identified EGFR mutations. Due to the absence of a considerable clinical benefit the gefitinib withdrawal from the market in the United States but in Europe and Asia is disposable.

The study which indicates that the presence of the EGFR mutation can play a critical role of choice of first line treatment in adenocarcinomas of the lung was the phase III Iressa Pan-Asia Study, or IPASS. [19, 20] In this trial participated patients with advanced NSCLC adenocarcinomas who previously were not treated and were never or light ex-smokers. These patients randomized to receive gefitinib versus carboplatin/paclitaxel. The group of patients who carried EGFR mutations and received gefitinib had shown very improved progression-free survival than those who received carboplatin/paclitaxel. On the other hand the patients who did not carried EGFR mutations they present longer progression-free survival when they treated with carboplatin/paclitaxel. Between the 2 groups there was not noticed significant benefit in overall survival.

Due to these results and to others from clinical trials, [21, 22] guidelines recommend to be tested all the patients with metastatic adenocarcinomas of the lung and those who carry an EGFR mutation to be treated in the first-line therapy with an EGFR-TKI.[5,6]. This happened on May 2013, when erlotinib was approved for first-line treatment of NSCLC for the patients whose tumors have EGFR exon 19 deletions or exon 21 (L858R) substitution mutations. Until then the TKI's were indicated to the $2^{\text {nd }}$ or to the $3^{\text {rd }}$ line of therapy.

Afatinib (Gilotrif) is a tyrosine kinase inhibitor that was approved in the United States in July 2013 for first-line treatment of patients with metastatic NSCLC whose tumors have EGFR exon 19 deletions or exon 21 (L858R) substitution mutations as detected by the diagnostic companion test, therascreen EGFR RGQ PCR Kit. Approval was based on data from the LUX-Lung 3 trial, comparing afatinib to chemotherapy with pemetrexed/cisplatin. Results showed the afatinib group's progression-free survival (PFS) was 11.1 months compared with 6.9 months for those treated with pemetrexed/cisplatin. Additionally, pa- 
tients with tumors expressing the 2 most common EGFR mutations (Del19 or L858R) taking afatinib lived over a year without tumor progression (PFS of $13.6 \mathrm{mo}$ ) compared with 6.9 months for those in the comparator arm. [23]

In contrast to the clear link between EGFR mutation status and EGFR-TKI response, the presence of EGFR mutations does not appear to predict response to treatment with the EGFR monoclonal antibody cetuximab. Data from the phase III First-Line Erbitux in Lung Cancer (FLEX) trial, which randomized patients to cisplatin/vinorelbine with or without cetuximab, showed that the presence of a mutation was not predictive of response to treatment. However, post hoc analysis showed that high expression of EGFR proteins on immunohistochemistry (IHC) was predictive of response to cetuximab plus chemotherapy vs chemotherapy alone. [24, 25]

An ongoing study evaluating the use of carboplatin and paclitaxel with or without bevacizumab and/or cetuximab in patients with metastatic or recurrent NSCLC will examine prospectively whether high EGFR protein expression can be used as a predictor of treatment response to cetuximab. Until then, the potential benefit of EGFR protein expression by IHC or EGFR gene amplification by fluorescence in situ hybridization (FISH) remains unclear. Cetuximab is FDA-approved for colorectal cancer and for head and neck cancer. It is not yet approved by the FDA for NSCLC.

Finally, the EGFR mutation T790M is found in approximately half of patients with acquired resistance EGFR-TKIs. [26] However, discontinuation of treatment can lead to a more rapid progression of disease regardless of T790M mutation status. [27] Because the presence of this mutation should not necessarily preclude continued use of EGFR-TKIs, the clinical relevance of routine testing in patients demonstrating treatment resistance is unknown.

Regarding the targeting of KRAS, no prospective trials have been conducted to demonstrate the potential value of testing for KRAS mutations and to tailor therapy accordingly. Although meta-analyses indicate that patients with KRAS mutations have a lowered response to EGFR-TKIs, most of the trials evaluated were small and it is unclear whether mutation status predicts reduced progression-free or overall survival. [28] Subgroup analysis of data from clinical trials evaluating EGFR-TKIs and cetuximab also showed no independent association between KRAS mutation status and survival. $[29,30]$ Nevertheless, despite the lack of definitive data demonstrating the benefit of KRAS mutation testing, the presence of mutations does seem to be associated with primary resistance to EGFR-TKI therapy. Testing can be considered to help in determining whether a patient might be a candidate for a TKI. [9]

Concerning the ALK targeting, the benefit of testing for ALK rearrangements was demonstrated in phase I and phtse II trials of the ALK inhibitor crizotinib. $[15,16]$ Results from these trials formed the basis of crizotinib's accelerated approval in the United States along with a companion diagnostic to evaluate ALK rearrangements on FISH. In these trials, patients with the EML4-ALK fusion, nearly all of whom had progressed despite at least 1 prior line of therapy, showed response rates of approximately $50 \%$ to $60 \%$ crizotinib. Response duration was $42-48$ weeks. [15, 16]

Because phase III trials are still underway and survival data are not yet available, researchers conducted a retrospective, nonrandomized analysis comparing patients enrolled in the 2 trials with historical controls to estimate the clinical benefit of crizotinib therapy. [31] In patients who received crizotinib as second-line therapy, the 1-year overall survival rate was $70 \%$ and the 2 -year overall survival rate was $55 \%$. By contrast, ALK -positive matched controls had a 1-year survival of $44 \%$ and a 2 -year survival of $12 \%$, whereas ALK -negative controls had a 1-year survival of $47 \%$ and a 2 -year survival of $32 \%$. These data suggest that the presence of the ALK gene fusion itself does not confer a poorer outcome but that the use of crizotinib in ALK -positive patients can improve outcomes. Based on these data, testing for ALK rearrangement is recommended in patients with metastatic NSCLC adenocarcinoma and the ALK inhibitor crizotinib is recommended for ALK-positive patients.

In April 2014, the FDA approved a second ALK tyrosine kinase inhibitor, ceritinib (Zykadia). It is indicated for ALK-positive, metastatic NSCLC in patients who have progressed on or are intolerant to crizotinib. The FDA granted ceritinib breakthrough therapy designation, priority review, and orphan product designation because of preliminary clinical evidence that the drug may offer a substantial improvement over available therapies. Early-stage results showed that ceritinib was highly active in patients with advanced, ALK-rearranged NSCLC, including those who experienced disease progression during crizotinib treatment, regardless of the presence of resistance mutations in ALK. [17] Ceritinib was shown to overcome crizotinib resistance mutations in a preclinical trial. In vitro and in vivo models of acquired resistance to crizotinib were evaluated, including cell lines established from biopsies of crizotinib-resistant NSCLC patients. Results revealed that ceritinib overcame crizotinib resistance mutations, in particular, ALK-harboring L1196M, G1269A, I1171T, 
and S1206Y mutations. [32]

Nowadays the new standard of care imposes to the stages IIIb and IV of lung adenicarcinomas DNA molecular testing for mutations in epidermal growth factor receptor (EGFR) and gene rearrangements of anaplastic lymphoma kinase (ALK). [33, 34]. There are 9 very large trials (Table 1, Optimal, First Signal, Ipass, Wjtog 340, Nejsg 002, Ensure, Eurtac, Lux-3, Lux-6) that show remarkable efficacy in patients with advanced lung cancer harboring EGFR mutations. In these studies the response rates oscillated in the impressive percentages from $55 \%$ to $86 \%$ and were connected with a remarkable median progression free survival of approximately 8 to 13 months, and with better quality of life compared to that of chemotherapy. These results are due to phenomenal effectiveness of gefitinib and erlotinib, the EGFR tyrosine kinase inhibitors (TKIs), against to the EGFR mutated tumours of NSCLC $[35,36]$, and of the crizotinib, the ALK TKI against NSCLC with ALK rearrangement. [37]. At this time there are many pivotal studies, for the earlier stages of NSCLC, that either are ongoing or in the process of developing yet, for such as these TKI's.

\section{Retrospective trials}

There are some small retrospective trials that they are looking about the role of TKI's at adjuvant treatment in NSCLC.
There is a retrospective analysis, from Memorial Sloan Kettering, with 167 patients, with completely resected stages I to III, of NSCLC, with EGFR mutation. Became a comparison between of a cohort of 56 patients to whom administered neoadjuvant or adjuvant EGFR TKI's and to a separate cohort of 111 patients to whom did not administered EGFR TKI's [38]. Subsequently a multivariate analysis showed that patients who treated with an EGFR-TKI presented improved 2-year disease free survival (DFS) rate of $89 \%$, thus the patients who did not treated with an EGFR-TKI the 2-year disease free survival (DFS) rate was $72 \%(\mathrm{P}=0.06)$. There was no statistical difference in overall survival. The 2-year overall survival was $\geq 90 \%$ in both groups. These results lead to conjecture that there may be a benefit and that had to be planned prospective analysis.

Table 1. EGFR TKI in stage IV

\begin{tabular}{llllll}
\hline Study & EGFR TKI & $\mathbf{n}$ & $\begin{array}{l}\text { Median PFS in } \\
\text { TKI arm } \\
\text { (months) }\end{array}$ & P value & HR \\
\hline OPTIMAL & Erlotinib & 154 & 13.1 & $<0.0001$ & 0.16 \\
First Signal & Gefitinib & 42 & 8.4 & 0.084 & 0.61 \\
IPASS & Gefitinib & 261 & 9.5 & $<0.0001$ & 0.48 \\
WJTOG 3405 & Gefitinib & 177 & 9.2 & $<0.001$ & 0.48 \\
NEJSG 002 & Gefitinib & 200 & 10.8 & $<0.001$ & 0.36 \\
Ensure & Erlotinib & 217 & 11 & $<0.0001$ & 0.34 \\
EURTAC & Erlotinib & 174 & 9.4 & $<0.0001$ & 0.42 \\
LUX-3 & Afatinib & 308 & 13.6 & $<0.0001$ & 0.47 \\
LUX-6 & Afatinib & 364 & 11.0 & $<0.0001$ & 0.28 \\
\hline
\end{tabular}

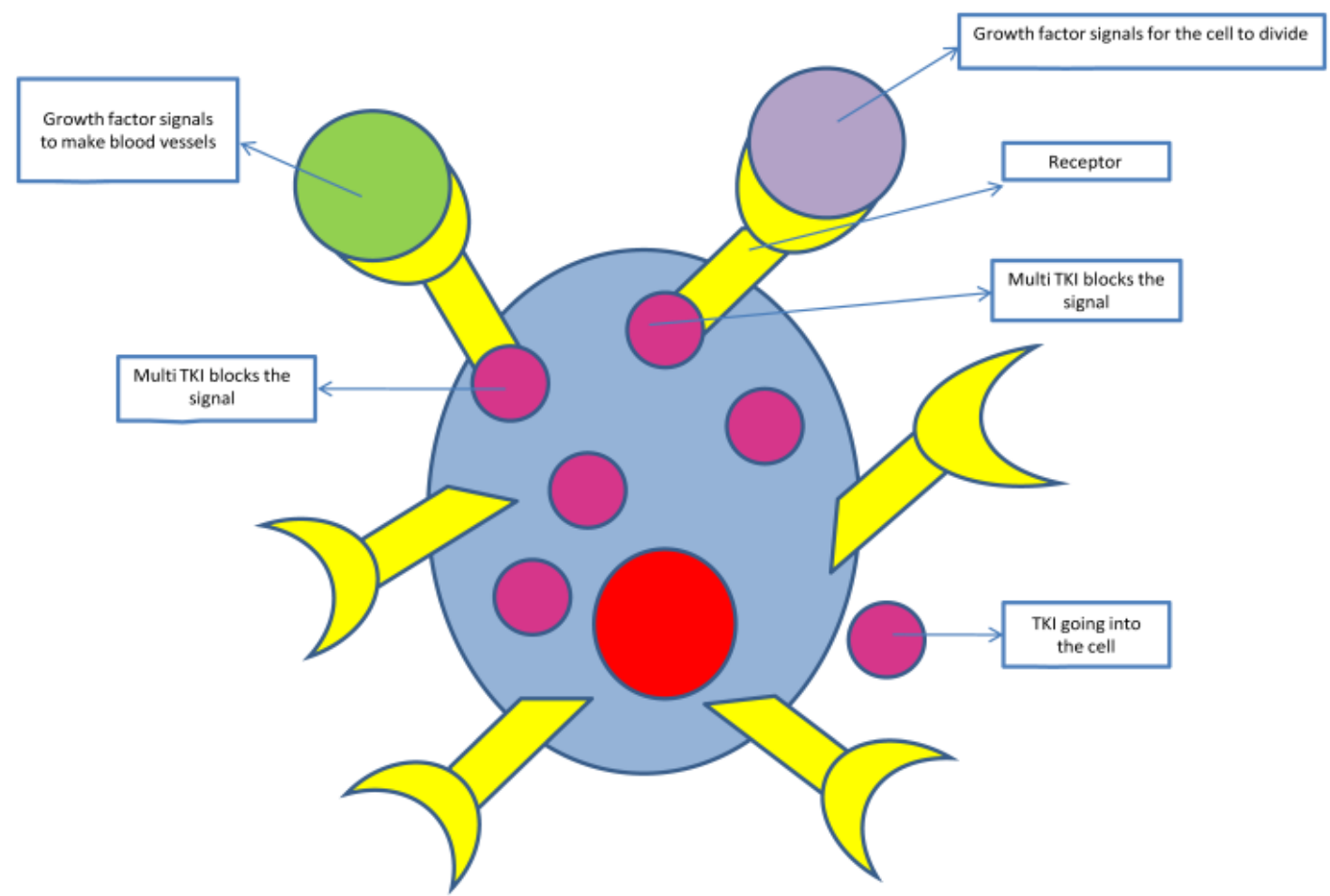

Figure 1: Tyrosine kinase inhibitors pathway blockage 
There are data from another retrospective study from Memorial, from 22 patients to whom administered treatment with EGFR TKI. To the 11 of them administered again treatment with TKI and 8 of them presented response for a median duration of 10 months [39]. It is noticeable that the tumours of the patients who underwent TKI therapy, was identified resistance mutation T790M. We can conclude from this, that as it happens to oncogenic addicted diseases, like breast cancer with positive estrogen-receptor, in which the 10 years adjuvant administration of tamoxifen improves the benefit from that of 5 years administration[40], or like to the Gastrointestinal stromal tumors (GISTs) in where the longer administration of imatinib gives better results. Survival was significantly improved for the group of patients treated with imatinib (gleevec) for 3 years compared to those treated for only one year. At 5 years, survival was $92 \%$ for the 3-year group and $81.7 \%$ for the 1-year group [41]. So and to the NSCLC, longer administration of adjuvant TKI therapy, may not improve overall survival but may increases the time to progression.

\section{Prospective trials}

There is also a small prospective trial of resected stage IIIA-N2, with EGFR mutated tumors that were randomized 1:1 to receive chemotherapy with pemetrexed and carboplatin for 4 cycles followed by gefitinib for 6 months versus chemotherapy only. In each arm randomized 30 patients, and it looked like the administration of gefitinib might potentially improve the progression free survival (40 versus 27 months, HR. 0.37; p=0.014;).Fig. 2

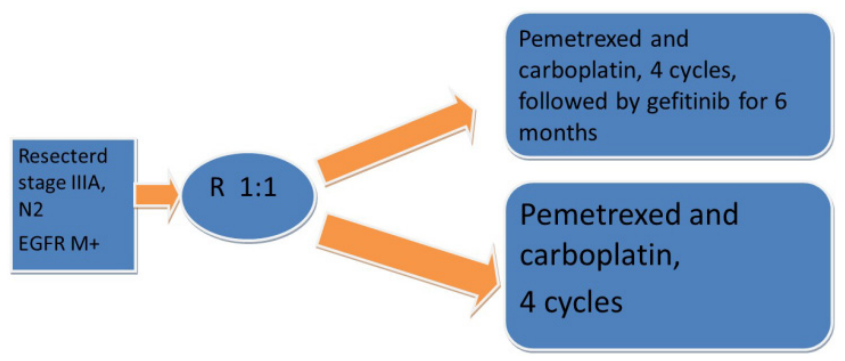

Figure 2: Prospectivephase II trial Adjuvant gefitinib in resected stage, IIIA, N2, EGFR M+

\section{BR.19}

There after we have some trials that are larger and they tried to find the role of TKI's in adjuvant setting (Table 2).

The BR.19 trial presented at ASCO at 2010. It is a trial in which participated patients with NSCLC, unselected molecularly, of resected stages IB to IIIA and randomized to receive after the completion of adju- vant chemotherapy according to the standard of care of each investigator, either adjuvant gefitinib for 2 years or only observation. But in 2005, due to the negative ISEL trial and to S0023 interim report, the enrollment of the patients stopped early, from a planned number of 1160 patients enrolled only 503.

The ISEL trial was stopped prematurely because failed to catch its overall survival endpoint. It was a trial for $2^{\text {nd }}$ line therapy in which patients, with stage IV of disease planned to receive gefitinib. [42]

To the phase III S0023 study participated patients of stage III of NSCLC, who underwent to concurrent chemo and radiotherapy, and randomized to be given gefitinib for more than 5 years or placebo. [43] But on 2005 an interim analysis showed that the patients who received gefitinib had 23 months of median survival time instead of patients who received placebo, who had 35 months $(\mathrm{p}=0.013)$. The analysis of this trial for the unselective population demonstrated that among the patients to whom administered gefitinib and to those to whom administered placebo there was not noticed any difference for the for disease free survival or for the overall survival. [44]. With respect to the patients whose tumours presented EGFR mutation, 40 of them who received placebo had a better overall survival than those, who were 36, who received gefitinib for the adjuvant treatment.

Another small phase II trial, a Chinese one, was presented at ASCO 2013, in which participated 60 patients of stage IIIA-N2 of NSCLC, molecurarly selected, with EGFR mutations, and who underwent in surgery, randomized to receive carboplatin/ pemetrexed for 4 cycles or carboplatin/ pemetrexed followed by administration of gefitinib for 6 months (45). The patients who received gefitinib had a better median DFS (39.8 vs. 27.0 mo, p=0.014, HR 0.37) and may be a trent for a better overall survival (41.6 vs. $32.6 \mathrm{mo}, \mathrm{p}=0.066$, HR 0.37). Due to the small number of patients who randomized to the trial, it is difficult to draw conclusions.

Table 2. Prospective trials: BR19, RADIANT, SELECT

\begin{tabular}{llll}
\hline & BR19 & RADIANT & SELECT \\
\hline Stage I & 50 & 51.0 & 45 \\
Stage IIA & 35 & 8.8 & 11 \\
Stage IIB & 35 & 20.6 & 16 \\
Stage IIIA & 15 & 17.6 & 28 \\
\hline
\end{tabular}

\section{SELECT}

There is a very good phase II, single arm trial, the SELECT. This study is the first one that focused on EGFR mutant tumors, and included patients with stage I to IIIA surgically resected. [46] The patients 
received standard-of-care adjuvant chemotherapy and/or radiation, depends on stage of disease, followed by adjuvant erlotinib at $150 \mathrm{mg}$ per os daily. This trial was subsequently expanded to 100 patients. The primary endpoint of this study was the DFS and the secondary endpoints were tolerability and safety and overall survival. After a median follow up time of 3.5 years, DFS is $89 \%$, median DFS has not yet be reached, above what was expected. The success of this trial is that $2 / 3$ of the patients received near 2 years of treatment. This means that went well followed up and well administered. Also important is that 24 patients have recurred, but only 2 of them during erlotinib treatment and the rest 22 after stop of erlotinibtreatment, in time of 1 to 2 years. The median overall survival has not yet been reached.

\section{RADIANT}

The very debate trial is the RADIANT trial which is a phase III trial. It was presented in ASCO 2014 in which targeted 973 patients. The stages of these patients was of stage I to IIIA of NSCLC and to their tumors the EGFR protein expression was positive, by immunohistochemistry (IHC), or obtained increased EGFR gene copy number by fluorescence in situ hybridization (FISH) [47]. Unfortunately the biomarker of this trial was not the presence of EGFR mutation. In this study patients randomized to receive erlotinib for 2 years versus placebo in 2:1. This trial stratified by histology, stage, adjuvant chemotherapy, smoking status, country, EGFR FISH status. For the unselected patient population the DFS shows no benefit (log-rank test: $p=0.3235$ ). For the subgroup of EGFR mutated tumors the $p$ value is significant but is not statistically significant due to hierarchical testing (log-rank test: $\mathrm{p}=0.0391)$.

\section{Discussion}

The questions that arise from this study where can we speak about reach cure with adjuvant treatment? The answer is not yet. There is benefit to DFS but not to OS. Is the number large enough to conclude about adjuvant benefit in this unplanned subgroup? The answer is probably not. Are we maybe modifying tumor biology? This question arises from the fact that $40 \%$ of patients from the erlotinib group presented CNS relapse. At this time we cannot give an answer to that and we have to investigate it more. Is the treatment exposure sufficient? Probably not because we know from other oncogene addicted diseases that the time of exposure is important, as it is mentioned earlier. Crossover to EGFR TKI jeopardizing observed benefit? This is not recommended because, as it is noticed from other trials the crossover affect the PFS.

\section{Future directions/conclusions}

This period are ongoing prospective trials in order to establish the benefit of adjuvant TKI's therapy, to the progression free survival and also to the overall survival in patients which are molecularly selected.

At this time, the NCI and cooperative oncology groups are planning 2 randomized trials of phase III, placebo-controlled, in the United States. To the one of them, participating patients who are EGFR mutant NSCLC and who after underwent surgery, randomizing to receive adjuvant erlotinib or placebo $(n=410)$. To the other one also participating patients with NSCLC, who underwent surgery, but to their tumours obtained ALK rearrangement $(n=336)$ [48]. The primary endpoint of these studies is the overall survival.

In Asia also are ongoing 2 randomized trials. In Japan to the WJOG6410L trial [49] participating patients with NSCLC, of stages II to III and randomizing to receive cisplatin/vinorelbine or gefitinib, after surgery $(n=230)$. In China to the ADJUVANT trial [50], also participating patients with NSCLC, of stages II to III, who also after surgery randomizing to receive cisplatin/vinorelbine or gefitinib $(n=220)$. The primary endpoint of the two Asiatic studies is the disease free survival.

Also is ongoing the phase II adjuvant afatinib trial. In this study are participating patients with NSCLC, whose tumors carry EGFR mutation, they underwent to complete surgical resection, of stages IA-B, IIA-B, IIIA-B. The patients randomized, 1 to 1 , to receive afatinib oral daily dose for 3 months or to receive afatinib for 2 years. The primary endpoint of this trial is the Recurrence-free survival and the secondary endpoints are the number of patients with adverse events, the molecular genotype of cancers with recurrence and the overall survival [51].

We hope that these trials will offer us the opportunity for a definitive answer of the role of tyrosine kinase inhibitors in induction treatment before chemo-radiotherapy or in early disease adjuvant therapy.

\section{Conflict of Interest}

None to declare.

\section{References}

1. Ansari J, Palmer DH, Rea DW, Hussain SA. Role of tyrosine kinase inhibitors in lung cancer. Anti-cancer Agents in Medicinal Chemistry 2009;9 (5): 569-75.

2. Kris MG, Natale RB, Herbst RS, et al. Efficacy of gefitinib, an inhibitor of the epidermal growth factor receptor tyrosine kinase, in symptomatic patients with non-small cell lung cancer: a randomized trial. JAMA. 2003;290:P2149-2158.

3. Posner I, Engel M, Gazit A, Levitzki A. Kinetics of inhibition by tyrphostins of the tyrosine kinase activity of the epidermal growth factor receptor and analysis by a new computer program. MolPharmacol. 1994;45 (4): 673-83.

4. Levitzki A, Mishani E. Tyrphostins and other tyrosine kinase inhibitors. Annu Rev Biochem 2006;75: 93-109. doi:10.1146/annurev.biochem.75.103004.142657. 
5. [Internet] National Comprehensive Cancer Network. NCCN Clinical Practice Guidelines in Oncology, Non-Small Cell Lung Cancer V2.2012. http://www.nccn.org/professionals/physician_gls/PDF/nscl.pdf.

6. Keedy VL, Temin S, Somerfield MR, et al. American Society of Clinical Oncology provisional clinical opinion: epidermal growth factor receptor (EGFR) Mutation testing for patients with advanced non-small-cell lung cancer considering first-line EGFR tyrosine kinase inhibitor therapy. J Clin Oncol. 2011;29(15):2121-7.

7. Stella GM, Scabini R, Inghilleri S, Cemmi F, Corso S, Pozzi E, et al. EGFR and KRAS mutational profiling in fresh non-small cell lung cancer (NSCLC) cells. J Cancer Res Clin Oncol. 2013;139(8):1327-35. doi: 10.1007/s00432-013-1444-y.

8. Sequist LV, Bell DW, Lynch TJ, Haber DA. Molecular predictors of response to epidermal growth factor receptor antagonists in non-small-cell lung cancer. J Clin Oncol. 2007;25(5):587-95.

9. Lynch TJ, Bell DW, Sordella R, et al. Activating mutations in the epidermal growth factor receptor underlying responsiveness of non-small-cell lung cancer to gefitinib. N Engl J Med. 2004;350(21):2129-39.

10. Pao W, Miller VA, Politi KA, et al. Acquired resistance of lung adenocarcinomas to gefitinib or erlotinib is associated with a second mutation in the EGFR kinase domain. PLoS Med. 2005;2(3):e73.

11. Kerr KM. Clinical relevance of the new IASLC/ERS/ATS adenocarcinoma classification. J Clin Pathol. 2013 Oct;66(10):832-8.

12. Shim HS, Lee da H, Park EJ, Kim SH. Histopathologic characteristics of lung adenocarcinomas with epidermal growth factor receptor mutations in the International Association for the Study of Lung Cancer/American Thoracic Society/European Respiratory Society lung adenocarcinoma classification. Arch Pathol Lab Med. 2011;135(10):1329-34.

13. Roberts PJ, Stinchcombe TE, Der CI, Socinski MA. Personalized medicine in non-small-cell lung cancer: is KRAS a useful marker in selecting patients for epidermal growth factor receptor-targeted therapy. J Clin Oncol. 2010;28(31):4769-77. doi: 10.1200/JCO.2009.27.4365.

14. Slebos RJ, Kibbelaar RE, Dalesio O, et al. K-ras oncogene activation as a prognostic marker in adenocarcinoma of the lung. $\mathrm{N}$ Engl $\mathrm{J}$ Med. 1990;323(9):561-5.

15. Kwak EL, Bang YJ, Camidge DR, et al. Anaplastic lymphoma kinase inhibition in non-small-cell lung cancer. N Engl J Med. 2010;363(18):1693-703.

16. Crins L, Kim D, Riely GJ, et al. Initial phase II results with crizotinib in advanced ALK-positive non-small cell lung cancer (NSCLC): PROFILE 1005. J Clin Oncol. 2011;29(suppl 15):Abstract7514.

17. Shaw AT, Kim DW, Mehra R, Tan DS, Felip E, Chow LQ, et al. Ceritinib in ALK-rearranged non-small-cell lung cancer. $N$ Engl J Med. 2014;370(13):1189-97.

18. Kris MG, Natale RB, Herbst RS, et al. Efficacy of gefitinib, an inhibitor of the epidermal growth factor receptor tyrosine kinase, in symptomatic patients with non-small cell lung cancer: a randomized trial. JAMA. 2003;290(16):2149-58

19. Mok TS, Wu YL, Thongprasert S, et al. Gefitinib or carboplatin-paclitaxel in pulmonary adenocarcinoma. N Engl J Med.2009;361(10):947-57.

20. Fukuoka $\mathrm{M}, \mathrm{Wu} \mathrm{YL}$, Thongprasert $\mathrm{S}$, et al. Biomarker analyses and final overall survival results from a phase III, randomized, open-label, first-line study of gefitinib versus carboplatin/paclitaxel in clinically selected patients with advanced non-small-cell lung cancer in Asia (IPASS). J Clin Oncol.2011;29(21):2866-74

21. Zhou C, Wu YL, Chen G, et al. Erlotinib versus chemotherapy as first-line treatment for patients with advanced EGFR mutation-positive non-small-cell lung cancer (OPTIMAL, CTONG-0802): a multicentre, open-label, randomised, phase 3 study. Lancet Oncol. 2011;12(8):735-42.

22. Rosell R, Gervais R, Vergnenegre A, et al. Erlotinib versus chemotherapy in advanced non-small cell lung cancer patients with epidermal growth factor receptor mutations: Interim results of the European Erlotinib Versus Chemotherapy (EURTAC) phase III randomized trial. J Clin Oncol. 2011;29(suppl 15):Abstract7503.

23. Sequist LV, Yang JC, Yamamoto N, O'Byrne K, Hirsh V, Mok T, et al. Phase III Study of Afatinib or Cisplatin Plus Pemetrexed in Patients With Metastatic Lung Adenocarcinoma With EGFR Mutations. J Clin Oncol. 2013;31(27):3327-34. doi: 10.1200/JCO.2012.44.2806. Epub 2013 Jul 1.

24. Pirker R, Pereira JR, von Pawel J, et al. EGFR expression as a predictor of survival for first-line chemotherapy plus cetuximab in patients with advanced non-small-cell lung cancer: analysis of data from the phase 3 FLEX study. Lancet Oncol. 2012;13(1):33-42. doi: 10.1016/S1470-2045(11)70318-7.

25. O'Byrne KJ, Gatzemeier U, Bondarenko I, et al. Molecular biomarkers in non-small-cell lung cancer: a retrospective analysis of data from the phase 3 FLEX study. Lancet Oncol. 2011; 12(8):795-805.

26. Gazdar AF. Activating and resistance mutations of EGFR in non-small-cell lung cancer: role in clinical response to EGFR tyrosine kinase inhibitors. Oncogene. 2009; 28 Suppl 1:S24-31.

27. Chaft JE, Oxnard GR, Sima CS, Kris MG, Miller VA, Riely GJ. Disease flare after tyrosine kinase inhibitor discontinuation in patients with EGFR-mutant lung cancer and acquired resistance to erlotinib or gefitinib: implications for clinical trial design. Clin Cancer Res. 2011; 17(19):6298-303.

28. Mao C, Qiu LX, Liao RY, et al. KRAS mutations and resistance to EGFR-TKIs treatment in patients with non-small cell lung cancer: a meta-analysis of 22 studies. Lung Cancer. 2010; 69(3):272-8.

29. Douillard JY, Shepherd FA, Hirsh V, et al. Molecular predictors of outcome with gefitinib and docetaxel in previously treated non-small-cell lung cancer: data from the randomized phase III INTEREST trial. J Clin Oncol. 2010;28(5):744-52.

30. O'Byrne KJ, Gatzemeier U, Bondarenko I, et al. Molecular biomarkers in non-small-cell lung cancer: a retrospective analysis of data from the phase 3 FLEX study. Lancet Oncol. 2011; 12(8):795-805.

31. Shaw AT, Yeap BY, Solomon BJ, et al. Impact of crizotinib on survival in patients with advanced, ALK-positive NSCLC compared with historical controls. J Clin Oncol. 2011; 29(suppl 15):Abstract7507.

32. Friboulet L, Li N, Katayama R, Lee CC, Gainor JF, Crystal AS, et al. The ALK inhibitor ceritinib overcomes crizotinib resistance in non-small cell lung cancer. Cancer Discov. 2014 Jun;4(6):662-73.

33. [Internet] http://www.tarceva.com

34. Frampton JE. Crizotinib: a review of its use in the treatment of anaplastic lymphoma kinase-positive, advanced non-small cell lung cancer. Drugs. 2013 Dec;73(18):2031-51.

35. Mok TS, Wu YL, Thongprasert S, et al. Gefitinib or carboplatin-paclitaxel in pulmonary adenocarcinoma. N Engl J Med 2009;361:947-57.

36. Rosell R, Carcereny E, Gervais R, et al. Erlotinib versus standard chemotherapy as first-line treatment for European patients with advanced EGFR mutation-positive non-small-cell lung cancer (EURTAC): a multicentre, open-label, randomised phase 3 trial. Lancet Oncol 2012;13:239-46.

37. Kwak EL, Bang YJ, Camidge DR, et al. Anaplastic lymphoma kinase inhibition in non-small-cell lung cancer. N Engl J Med 2010;363:1693-703.

38. Janjigian YY, Park BJ, Zakowski MF, et al. Impact on disease-free survival of adjuvant erlotinib or gefitinib in patients with resected lung adenocarcinomas that harbor EGFR mutations. J ThoracOncol 2011;6:569-75

39. Paez JG, Jonne PA, Lee JC, et al. EGFR mutations in lung cancer: correlation with clinical response to gefitinib therapy. Science 2004:304:1497-500.

40. Gray RG, Rea D, Handley K, et al. aTTom: long-term effects of continuing adjuvant tamoxifen to 10 years versus stopping at 5 years in 6,953 women with early breast cancer. J ClinOncol 2013;31:abstr5.

41. Joensuu $\mathrm{H}$, et al. One vs three years of adjuvant imatinib for operable gastrointestinal stromal tumor: a randomized trial. JAMA. 2012;307(12):1265-72. doi: 10.1001/jama.2012.347

42. Thatcher N, Chang A, Parikh P, et al. Gefitinib plus best supportive care in previously treated patients with refractory advanced non-small-cell lung cancer: results from a randomised, placebo-controlled, multicentre study (Iressa Survival Evaluation in Lung Cancer). Lancet 2005;366:1527-37.

43. Kelly K, Chansky K, Gaspar LE, et al. Phase III trial of maintenance gefitinib or placebo after concurrent chemoradiotherapy and docetaxel consolidation in inoperable stage III non-small-cell lung cancer: SWOG S0023. J ClinOncol 2008;26:2450-6

44. Goss GD, O'Callaghan C, Lorimer I, et al. Gefitinib versus placebo in completely resected non-small-cell lung cancer: results of the NCIC CTG BR19 study. J ClinOncol 2013:31:3320-6.

45. Wang SY, Ou W, Li N, et al. Pemetrexed-carboplatin adjuvant chemotherapy with or without gefitinib in resected stage IIIA-N2 non-small cell lung cancer harbouring EGFR mutations: a randomized phase II study. J ClinOncol 2013:31:abstr7519.

46. Neal JW, Pennell NA, Govindan R, et al. The SELECT study: a multicenter phase II trial of adjuvant erlotinib in resected epidermal growth factor receptor (EGFR) mutation-positive non-small cell lung cancer (NSCLC). J ClinOncol 2012;30:abstr7010

47. Coleman BK, Curtis LH, Onaitis MW, D'Amico TA, Berry MF. Adjuvant chemotherapy after resection of N1 non-small cell lung cancer: differential impact of new evidence on physician and patient decisions. J Thorac Dis. 2015 Mar;7(3):243-51

48. Govindan R. Lung Cancer: Molecular Profiling and Genomics (Eductation Session Presentation). ASCO2013.

49. Tada H, Takeda K, Nakagawa K, et al. Vinorelbine plus cisplatin versus gefitinib in resected non-small cell lung cancer haboring activating EGFR mutation (WJOG6410L) J ClinOncol 2012;30:TPS7110.

50. Wang Y, Chen J, Wu S, et al. Clinical effectiveness and clinical toxicity associated with platinum-based doublets in the first-line setting for advanced non-squamous non-small cell lung cancer in Chinese patients: a retrospective cohort study. BMC Cancer. 2014 Dec 12;14:940.

51. [Internet] http://www.clinicaltrials.gov/ct2/show/NCT01746251?term= NCT01746251\&rank= 